\title{
Outline of Calendar Studies Conducted at the Byurakan Astrophysical Observatory in 1983-2021
}

\author{
H.A. Malkhasyan * \\ NAS RA V. Ambartsumian Byurakan Astrophysical Observatory (BAO), Armenia
}

\begin{abstract}
The calendar studies conducted at the Byurakan Astrophysical Observatory in 1983-2021 are the result of Grigor Broutian's initiation and scientific activity. The approaches used by him have raised a number of new issues and provided extremely important results that are a serious incentive for the future development of this multilayered and multifaceted field in terms of astronomy and historical culture.

Almost all the problems of the Armenian and adjacent calendars of the Christian period have been discussed and finally resolved. The Armenian calendar attributed to Hayk Nahapet has been thoroughly researched and brought to an astronomically grounded comprehensive solution. The preliminary, so-called Protohaykian calendar has been revealed with its main and key aspects, and received astronomical as well as archaeological real evidence. All in all, in general the 11000year-old Armenian calendar-astronomical traditional historical culture has been deciphered in its continuity. Some new data have been obtained in favor of the existence of an earlier, the so-called "Initial Calendar" as well. Moreover, common close relations have been revealed in the field of natural-philosophical-religious and their historical-cultural-astronomical traditional perception of the creation of the ancient Armenian "Vishap" ("dragon") stone monuments, the "Zorats Qarer" megalithic ensemble, the "Sasnay Tsrer" (Daredevils of Sassoun) epic poem, the Armenian folk tales and archaeological materials.
\end{abstract}

Keywords: Armenian Calendar History: Archeoastronomy: Grigor Broutian: Zorats Qarer: Virgo: Haykian Calendar: Protohaykian Calendar: Cultural astronomy: Armenian folklore:

\section{Introduction}

Archaeoastronomy and Cultural Astronomy department of the Byurakan Astrophysical Observatory was created in 2017 under the supervision of an astronomer, calendar scientist, $\mathrm{PhD}$ in phys.math. sciences Grigor Broutian. Until then, from the 60-70s, H. Badalian's and B. Tumanyan's research and monograph $^{1}$ in the field of calendar studies were known. Separate works of E. Parsamian on the archaeological site of Metzamor Astronomical Platform ${ }^{2}$ and "Zorats Qarer" monument ${ }^{3}$ were printed as well. Unfortunately, the mentioned period is not covered further in this paper and requires a complete

\footnotetext{
${ }^{*}$ hayk_malkhasyan@bao.sci.am

${ }^{1}$ Tumanyan B., History of Armenian Astronomy, vol. 1. "Mitq", Yerevan, 1964 (in Armenian)

Tumanyan B., Manual Calendar, Acad. Sci. of ARM SSR Publishing-House, Yerevan, 1965 (in Armenian)

Tumanyan B., History of Armenian Astronomy, vol. 2. "Mitq", Yerevan, 1968 (in Armenian)

Badalian H., History of Calendar, Acad. Sci. of ARM SSR Publishing-House, Yerevan, 1970 (in Armenian)

Tumanyan B., History of Calendar, "Mitq", Yerevan, 1972 (in Armenian)

Badalian H., History of Armenian Calendar, Acad. Sci. of ARM SSR Publishing-House, Yerevan, 1976 (in Armenian)

Tumanyan B., Mirzoean L., Astronomy, Yerevan University Publishing House, Yerevan, 1978 (in Armenian)

Tumanyan B., History of Armenian Calendar, Yerevan University Publishing House, Yerevan 1985 (in Armenian)

${ }^{2}$ Parsamian E., Astronomical Significance of the platforms of Small Hill, Khanzadian E.V., Mkrtchian K.H., Parsamian E.S., Metsamor, Acad. Sci. of ARM SSR Publishing-House, Yerevan, 1973, pp.142-149 (in Armenian)

${ }^{3}$ Parsamian E.S., On Possible Astronomical Significance of Megalithic Rings of Angelacot. Communications of BAO, 1985, vol. 57, pp. 101-103.
} 
separate research and presentation. Thus, only the first research period of Gr. Broutian's calendar studies will be discussed here (1983-2021).

In modern life, the calendar is viewed merely as an instrument for time measurement. In our daily lives we clarify the date and check digital watches for unlimited times, while until the past centuries the traditional use of sundials was preserved in Armenia. The principle of the latter's operation, as we know, is the shadow change during the Sun's daily rotation period. This is a simple argument in favor of the fact that the ancient Armenian (as well as of other nations) calendars must have had the observational basis ${ }^{4}$.

Unfortunately, we have no information about Armenian calendars of pre-Christian times, however, Armenian medieval bibliographic material of the Christian period is quite rich with data on calendars. Meanwhile, our knowledge in only calendars used through the rich history of Armenia is not enough to get a clear idea on their origin, structural features, changes and religious and cultural manifestations. In general, to form a general understanding about any calendar the following questions ${ }^{5}$ should at least be answered:

1) When was the calendar founded (and by whom)?

2) What structure did it have?

3) When did the years count start? (what's the inception of the calculations)

4) What changes did it undergo later (when and who was the initiator)?

The answers to these quite important questions were sought in the historical-cultural studies of the Byurakan Astrophysical Observatory during the last decades.

These studies were conducted in "reverse chronological" sequence taking Bibliographic heritage of the Middle Ages as an inception. In natural sciences this research method is known as a "reverse

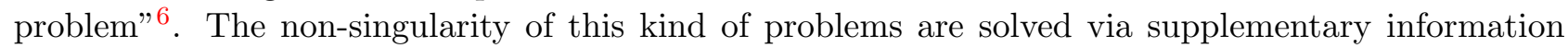
that can often come from completely other spheres (Folklore, Ethnography, Agriculture, Archeology, Mythology, etc.).

In this regard, it would be natural to present the results of the research on the Armenian calendars in the reverse chronology as well (from the most recent times to the early and "ancient" millennia). Conditionally, we will divide the calendar history into two periods: Christian and pre-Christian, considering $301 \mathrm{AD}$ (the introduction of Christianity in Armenia) as the transition point. For this kind of "chronological chain" the calendar, its issues and "pillars" of the research will be discussed separately to present the results of calendar studies in a logical and a simplified manner possible. The obtained results will be provided in the form of answers to the 4 questions listed above.

\section{Period of Christianity}

It is known, that prior to $553 \mathrm{AD}$, the 200 year-long chart created by Andreas Byzantine was used to determine Christian holidays, while before that 95-year long holiday calendars of Ghevondes, Vorogines and Anatolios were used. These all were composed according to a fixed, non-Armenian calendar system like the Julian calendar. Only in the mid. $6^{\text {th }}$ century, when the above-mentioned Byzantine's lit of Eater dates came to its end, the Armenian church aimed at establishing independent church calendar, and resulted in Armenian Major Calendar.

\footnotetext{
${ }^{4}$ Gr. Broutian, On Some Questions about Armenian Calendar, the Armenian Calendar and Celestial Bodies, "Etchmiadzin", 1995, Issue 1, pp. 47-60 (in Armenian)

${ }^{5}$ Gr. Broutian, The Armenian Calendar from Its Foundation to the Present, NAS RA Byurakan Astrophysical Observatory, Yerevan, "Yanos", 2018, p.21 (in Armenian)

${ }^{6}$ Ambartsumian V., The reverse problems in the natural science, Acad. Sci. of ARM SSR Publishing-House, Yerevan, 1983
} 


\subsection{Armenian Major Calendar}

In the Middle ages, until the $19^{\text {th }}$ century, the Armenian Major Calendar ${ }^{7}$ was widely used in Armenia. Although the information about this calendar is relatively rich, the answers to the foregoing questions are often contradictory among the authors of the 10-11 th centuries. The foundation of this calendar is connected to the names of three different Catholicoses, thus dates differ. Later to overcome these difficulties modern researchers presented the foundation of this calendar in two stages: the establishment process itself and its official verification. The questions on its structure also received two different and contradictory answers. Some authors considered the Armenian Major Calendar fixed, while others argued it being a calendar of 365 days with movable beginning of the year. There are also two different beliefs on the beginning of the years count July 11, 552 AD and a year later (553 AD). The situation is clearer in regards to the changes of this calendar, in which case the opinions mostly coincide. Almost all agree that the Armenian Major Calendar was reformed by Anania Shirakouni (Shirakatsi) in the $7^{\text {th }}$ century.

To clarify disagreements of this kind we conducted in depth studies of the calendar tables authored by Anania Shirakouni, namely Revelation and Easter addresses (532-year tables), Hovhannes Sarkavag's calendar copy, The interpretation of the Armenian calendar, etc. Main results are obtained primarily due to the analysis of these works, and presented as a sequel of papers ${ }^{8}$.

Below you can find the results on the Armenian Major Calendar in the form of answers to the above-mentioned questions:

1) It was founded by the order of Armenian Catholicos Movses Eghivandetsi in 552 AD. Athenas Taroneci was the author.

2) The duration of the year was always 365 days without leap years. A year, i.e. 360 days (12x30) and additional 5 days (Aveleac). With the movable beginning of the year with Navasard ${ }^{9} 1$.

3) According to Athenas the beginning of the years count was July 11, $552 \mathrm{AD}$, while according to the reforms made by Anania Shirakouni it was on January 6, 553 AD (with fixed start of the year).

4) It was reformed by Anania Shirakouni in 666-667 by the order of Anastas A (1st) Akoreci catholicos ${ }^{10}$. The beginning of the year was separated from Navasard 1 and was fixed prior to January 6 .

Next reform of the Armenian calendar was made by Hovhannes Sarkawag in the $12^{\text {th }}$ century and this new calendar: Armenian Minor Calendar was put into practice.

\footnotetext{
${ }^{7}$ In the literataure on Calendar studies this calendar is known by different names: The Armenian Date, Haykazian Date, The Main Armenian Date, Asqanazian Date etc.

${ }^{8}$ Gr. Broutian, On Some Questions about Armenian Calendar: Armenian Major Calendar, "Etchmiadzin", 1983, Issue 8, pp.41-44 (in Armenian)

Gr. Broutian, On Some Questions about Armenian Calendar: the Armenian Calendar until Armenian Major Calendar, "Etchmiadzin", 1984, Issue 5, pp.54-58 (in Armenian)

Gr. Broutian, On Some Questions about Armenian Calendar: the Improvement of the Armenian Calendar and the List of Catholicoses with Dates, "Etchmiatdzin", 1994, Issue 8, pp.30-48 (in Armenian)

Gr. Broutian, The Circle 532 of Anania Shirakouni According to the Ancient Armenian Paper Manuscript, "Etchmiadzin", 1998, Issue 1, pp.125-143 (in Armenian)

Gr. Broutian, "Kharnakhoran" by Anania Shirakouni, Ed. Art. Matevosyan, Mother See of Holy "Etchmiadzin", 1999 (in Armenian)

Gr. Broutian, About a new component of the calendar of Anania Shirakouni, "Bazmavep", 2011, vol. 1-2, pp.181-215 (in Armenian).

Gr. Broutian, The Construction of "Tomar" (the Calendar) of Anania Shirakouni, "Bazmavep", 2013, vol. 3-4, pp.11-27 (in Armenian)

Gr. Broutian, The Construction of Calendars of Anania Shirakouni and Yovhannes Sarkawag and Their Relation, "Bazmavep", 2014, vol. 3-4, pp.11-24 (in Armenian)

${ }^{9}$ Gr.Broutian, On Some Questions about Armenian Calendar. The Main Armenian Holiday, "Etchmiadzin", 1995, Issues 10-12, pp.130-153 (in Armenian)

${ }^{10} \mathrm{Gr}$. Broutian, On the circumstances of the creation of Anania's K'NNIKON, "Bazmavep", 2016, vol. 1-2, pp.11-30 (in Armenian)
} 


\subsection{Armenian Minor Calendar}

The fixed calendar created by Hovhannes Sarkawag is known as "Sarkawagadir Karg" 11 (Discipline of Sarkawag) ${ }^{12}$. It's in detail explanation can be found in calendrical "PATCHEN" ("The Copy"), and there are two main works related to it: Editing of "KHARNAKHORAN" by Sarkawag by Anania Shirakouni and famous calendar tables titled "Haysmavoracuyc" by Hovhannes Sarkawag. Unlike Anania Shirakouni, who in order to fix the calendar separated the start of the year from Navasard 1 and placed it on January 6, Sarkawag introduced a leap day to the Armenian calendar for the first time seeking the same purpose, which supplements 365 days of the Armenian calendar every 4 years. For a long time, there was an opinion in the Armenian calendar studies that Sarkawag added the leap day to the end of the year, after 5 days that are called Aveleac, however, it's not evidenced by any fact. The comprehensive research of Sarkawag's calendar studies reveals information on the position of the leap day. In "KHARNAKHORAN" edited by Hovhannes Sarkawag, in front of March 8 prior to Month Areg it's written, "Hayq (the Armenians) place the unnecessary leap day here". In the paper of "PATCHEN TOMARI" (The Copy of the Calendar) it says, "The leap year's month of Mehekan is 31...". This means that in "Sarkawagadir Karg" the leap day is placed between the months of Mehekan and Areg (before March 8).

Apart from fixation, Sarkawag changed the position of the start of the year as well, placing it on August 11. This change is probably the most discussed one by calendar scientists. They attempted to explain it as the main day of the start of the year in the Armenian calendar of the ancient times, and connect it to Hayk Nahapet's deeds: the victory over Bel and etc. Nevertheless, Sarkawag explained in his papers the reason he chose August 11 as the start of the year. He mentioned that the translations of the Bible and of some other books done in the beginning of the $5^{\text {th }}$ century served as a basis. Even during the first translations of these books the movable start of the year coincided with August 11. Having no other principle or an inception point except the date comparisons in the above-mentioned translations and seeking to fix the calendar, Hovhannes Imastaser chose the start of the year so that the letter was in harmony with the existing dates. Hence, it should be noted that this is a common Roman date to which coincided the Armenian movable start of the year: Navasard 1, 428-431 AD. August 11 has no other significance other than this, and giving it another meaning or definition is wrong.

All in all, as a result of the study of the Armenian Minor Calendar we learn that:

1) It was founded by the Armenian Catholicos Gregory II Vkayaser and compiled by Hovhannes Sarkawag in 1112 AD.

2) The medium duration of the year was 365,25 days $(12 \times 30$, Aveleac 5 days and the added leap day every four years). The leap day was placed between the months of Mehekan and Areg before March 8 (every 4 years Mehekan lasted 31 days).

3) The beginning of the years count was August 11 (Navasard 1) $1084 \mathrm{AD}$ with fixed start of the year.

4) It didn't undergo changes.

Though the Armenian Minor Calendar had limited use, it was applied as a church calendar till the $19^{t h}$ century. Parallel to it already in the $18^{t h}$ century the Roman calendar was put into practice.

\section{Pre-Christian Period}

We haven't received any direct evidence of the Armenian calendar of the pre-Christian period. We can understand the nature of that calendar by analysing the structure of the Armenian traditional calendar and other realities associated with the calendar.

\footnotetext{
${ }^{11}$ This Calendar is also known as "Mtruk"

${ }^{12}$ Gr. Broutian, On Some Questions about Armenian Calendar. Armenian Minor Calendar (Sarkawagadir Calendar), "Etchmiadzin", 1994, Issues 4-5, pp.100-116 (in Armenian)

Gr. Broutian, The Chronological System of Hovhannes Sarkawag, "Magaghat" Publishing House, Yerevan, 1999. (in Armenian)

Malkhasyan H.A. doi: https://doi.org/10.52526/25792776-2021.68.2-407 


\subsection{Armenian Haykian Calendar}

According to medieval calendar scientists, the foundation of the Armenian calendar is associated with Hayk Nahapet. It's also known that the Armenians named one of the brightest constellations, the famous Orion, in the name of Hayk. The analyses of the various data indicate that the heliacal rising ${ }^{13}$ of the main Betelgeuse star ("Hayk's Eastern Shoulder", $\alpha$ Orionis) of this constellation had close connection to the Armenian Holiday of Navasard. Thus, it becomes possible to specify the time of this astronomical phenomenon and combine it with the already known historical data. The calculations have shown that from the center of Armenian Highland (39, $5^{\circ}$ latitude North) the heliacal rising of the above-mentioned star was seen in the period of summer solstice, 8 days prior to it to be more precise. Conducting a calendar countdown and comparing it to the well-known historical events, it became known that the beginning of the Haykian calendar is 2341 BC. This date was grounded quite well ${ }^{14}$. This is evidenced by detailed analyses of many bibliographic, historical, mythological, ethnographic, archaeological and other realities. In particular historical facts known from the Cuneiform sources were compared to the information Armenian historians conveyed. Complete parallels and stylized canonical identifications ${ }^{15}$ were obtained between the story of Hayk and Bel and the conflict between Sargon (I) the Great, ruler of northern Mesopotamia, and Lugalzagesi, the last ruler of the Early Dynastic period of Sumer.

What is generally known about the Haykian Calendar?

1) It was founded in 2341 BC by Hayk Nahapet (most probably by Sargon I the Great)

2) It was composed of 12 months of 30 days (360 days) and additional 5 days, which were called Aveleac. The start of the year was movable. The fixed holiday of Navasard was celebrated by the observation of the heliacal rising of the Betelgeuse star of Hayk constellation (Hayk's Eastern Shoulder, $\alpha$ Orionis).

3) The years count began in 2341 BC, 8 days prior to summer solstice, June 14 (Navasard 1).

4) Most probably it didn't undergo any changes and was also parallely used ${ }^{16}$ after the introduction of Christianity until $552 \mathrm{AD}$.

It is natural that before Hayk Nahapet's arrival to the country of Ararat from Babylon, the inhabitants of the land should have had their culture, the inseparable part of which is the calendar. It was conditionally called Protohaykian calendar considering its usage before Hayk Napahet's arrival.

\subsection{Protohaykian Calendar}

Many medieval authors (Anania Shirakouni, Hovhannes Sarkawag, Grigor Tatevatsi, Hovhannes Vanakan (Monk) et al.) conveyed that months of the Armenian calendar were named after Hayk's children. All of them mention that Hayk had 10 children and only 10 months bore their names. The other 2 months were called "i gortsoc" ("of works") or "i veray yeghanakacd" ("on top of the seasons"). The study of the above-mentioned sources on Hayk's 10 children has shown that Hayk had 3 sons and 7 daughters. This coincides with the fact that the main star of the Hayk constellation was seen 295 days, while it was beneath the horizon and invisible during the other 70 days. These 295 days correspond to the sum of 10 lunar months (10 x 29,5 days). This fact (also other supporting materials) allow us to conclude that the main year in Hayk's calendar consisted of 10

\footnotetext{
${ }^{13}$ Gr. Broutian, Relations of the Oldest Armenian Calendars with Hevenly Bodies, "Bazmavep", 2017, vol. 1-2, pp.39-59 (in Armenian)

${ }^{14}$ Gr. Broutian, On Some Questions of Armenian Calendar: The Main Armenian Date, "Etchmiadzin", 1985, Issue 1, pp.51-57 (in Armenian)

Gr. Broutian, On Some Questions of Armenian Calendar: Analysis of 2341 BC, "Etchmiadzin", 1985, Issues 2-3, pp.72-80 (in Armenian)

Gr. Broutian, The Armenian Calendar, "Etchmiadzin", 1997, Chapter 7, pp.211-246 (in Armenian)

${ }^{15} \mathrm{Gr}$. Broutian, On Some Questions of Armenian Calendar: the beginning of the Armenian Main Calendar and Historical Facts, "Etchmiadzin", 1997, Issues 2-3, pp.144-155 (in Armenian)

${ }^{16}$ Gr. Broutian, On Some Questions of Armenian Calendar: the Structure of the Protohaykian Calendar, "Etchmiadzin", 1996, Issue 12, pp.135-164 (in Armenian)
} 
months, the remaining 65-70 days were considered out of the year, as the main star of Father God was not visible in the sky. This structure ${ }^{17}$ of Protohaykian calendar was later approved by the analysis ${ }^{18}$ of the archaeological material ((ornaments of the black polished ware (the end of IV millennium BC) excavated from Keti archaeological site, Shirak Region, Republic of Armenia). It was also discovered that the canonical duration of the calendar was not 295, but 300 days, and the 5-day difference was meantime considered as a part of the year and separate.

This calendar impacted every aspect ${ }^{19}$ of Armenian culture (as well as of other nations). We have received only fragments in the materials of folklore, ethnography, historiography and archeology. The study and analysis ${ }^{20}$ of all these has revealed that the Protohaykian calendar is closely related to cereal cultivation (agriculture). Such an extensive analysis has led us to conclude that the main star of this calendar was the brightest Spica ( $\alpha$ Virginis) in the constellation Virgo. In the Sumerian astronomical catalog, the name of this star ${ }^{m u l} \mathrm{AB} \cdot \mathrm{SIN}_{2}$ is translated as "groove, ridge" and is linked ${ }^{21}$ to the culture of agriculture and the name of the virgin goddess Sillia. And the observation of the heliacal rising of this very star, the start of the year, the main Armenian holiday Navasard, was celebrated. The most significant event in farmers' lives was the autumn cereal harvest, which takes place close to summer solstice. Therefore, it was not difficult to calculate in what year the heliacal rising of the main star of the Virgo constellation preceded the summer solstice for 8 days. This revealed the beginning ${ }^{22}$ of the Protohaykian calendar, which is $9000 \mathrm{BC}$ (with a possible mistake of 80 years). It is interesting that the same year was obtained by the astronomical examination ${ }^{23}$ of one of the platforms of the ancient site of Metzamor as well. By this it has also been found out that heliacal rising of Pleiades was most probably observed in 9000 BC. Very recently this date was approved during the astronomical study ${ }^{24}$ of "Zorats Qarer" megalithic monument. We discovered that on one of the central stones ( $N$ 198) there is a specially cut corner the direction of which corresponds to the point of the sky, by which the Spica star of Virgo constellation $(\alpha$ Virginis) passed on summer solstice right before sunrise in 9000 BC (Figure 1). The dates obtained in two different ways are in accord, which can be considered as an exceptionally accurate result for such ancient times. Due to its respectable antiquity, the Protohaykian calendar should be the subject of further studies as well. We do not have the opportunity to discuss historical figures and events in detail here, simple because we have no direct evidence of them. So, let's see what is already known and what is yet to learn about this calendar.

1) It was founded in $9000 \mathrm{BC}$ (with possible mistake of 80 years) by the unknown farmers.

2) It's composed of the main year of 300 days and 65-70-day out of the year period. The start of the year is relatively fixed ( 1 day in about 100 years due to the Earth's precession and the angular velocity of the star's proper motion) 8 days prior to the summer solstice (Navasard 1 June 14). 5 out of 300 days of the year were considered both as part of the year and out of it. Structurally it is a lunisolar calendar.

3) The year count started probably at the same time.

\footnotetext{
${ }^{17}$ Gr. Broutian, Ancient Armenian Calendar Ideas According to "Armenian History" by Agatangeglos, "Etchmiadzin", 1998, Issue 6, pp.45-53 (in Armenian)

${ }^{18}$ Gr. Broutian, The Oldest Armenian Calendar Concept According to the Analyze of the Ornaments of a Vessel from 28-27 ${ }^{\text {th }}$ c. B.C., "Bazmavep", 2007, pp. 149-163 (in Armenian)

Gr. Broutian, The Armenian Calendar of the Early Bronze Age According to Ornaments of the Black Polished Ware of Keti, 254 pages (unpublished monograph)

${ }^{19}$ See as an example: Gr. Broutian, The Concepts of the Oldest Armenian Calendar in Greek Epic Poem about Basil Digenis Akritas, "Bazmavep", 2004, vol. 3-4, pp.5-16 (in Armenian)

${ }^{20}$ Gr. Broutian, The Beginning of the Protohaykian Calendar, "Bazmavep", 2016, vol. 3-4, pp.11-63 (in Armenian)

${ }^{21}$ Davtyan A., Armenian Stellar Mythology, Yerevan, 2004, pp.158-161 (in Armenian)

${ }^{22}$ Gr. Broutian, The Beginning of Protohaykian Calendar, Non-stable Universe: Energetic Resources, Activity Penomena, and Evolutionary Processes, Proceedings of an International Symposium dedicated to the 70th anniversary of the Byurakan Astrophysical Observatory held at NAS RA, Yerevan and Byurakan, Armenia 19-23 September 2016, Edited by A. Mickaelian, Astronomical Society of the Pacific, Conference Series, vol. 511, San Francisco, 2017, pp. 296-302

${ }^{23}$ Gr. Broutian, "Bazmavep", 2017, Ibid.

${ }^{24}$ Gr. Broutian, H. Malkhasyan, About Some Preliminary Results Based on the Last Measerments of "Zorats Qarer" Megalithic Monument, "Bazmavep", 2021 (accepted)
} 


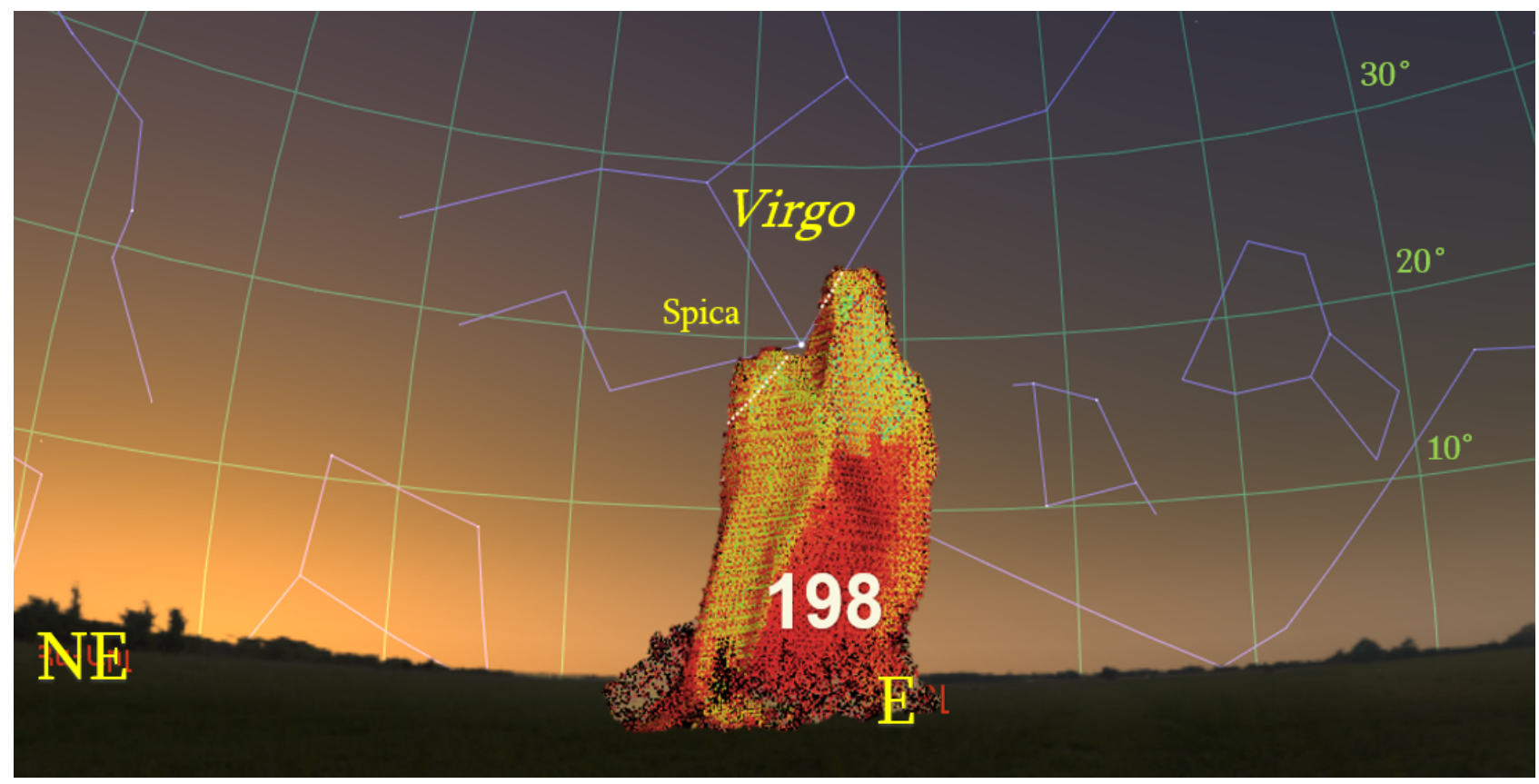

Figure 1. The megalithe with special angle N 198 (the direction of the angle - Azimuth $=262^{\circ} 50^{\prime}$ from the South point, Elevation $=19^{\circ} 34^{\prime}$ ). "Zorats Qarer" megalithic monument (Armenia, $39^{\circ} 33^{\prime}$ latitude North), 9000 BC (the beginning of Armenian Protohaykian Calendar). Summer solstice, before sunrise. Reconstructed in Stellarium v0.20.4 (http://www. stellarium.org).

4) There is a strong doubt that this calendar underwent changes in the first half of the VI millennium BC $^{25}$. The observed main star, Spica ( $\alpha$ Virginis), was replaced by Sirius ${ }^{26}$ ( $\alpha$ Canis Majoris) to keep the start of the year fixed. There is no information about the structural or any other changes of the mentioned calendar yet.

As we see, the structure of the Protohaykian calendar is quite complex. Despite its extreme antiquity, this kind of structural complexity implies that it must have developed for millennia.

\subsection{The Preliminary Calendar}

What was the calendar like before the foundation of the Protohaykian calendar? For now, we can only have hypotheses and suggestions. However, the existence of a calendar prior to the foundation of the Protohaykian cannot be ruled out. To this one the term Preliminary Calendar ${ }^{27}$ is applied. Nevertheless, our knowledge of the latter is very poor. Thus, the lack of answers to the above 4 questions implies the continuation of the study in this area. Entirely convinced of the need for further research of the field, Gr. Broutian developed and published a scientific-methodological manual ${ }^{28}$ dedicated to the basic knowledge and research methodology required for the research of the Armenian calendars.

In the table 1 you can find the calendars that were used in Armenia and discussed in this paper with their application periods ${ }^{29}$.

It is obvious from the table that the Protohaykian calendar is "the most longevous" of all presented (66.5 centuries).

\footnotetext{
${ }^{25}$ Gr. Broutian, H. Malkhasyan, Ibid.

${ }^{26}$ It should be noted here that in Mesopotamian sources this star ${ }^{m u l}$ GAG.SI.DI is translated as "arrow", and it's interesting that there is a deity named "Arrow" in the Sumerian sources, who guards the doors of the underworld. See for example Davtyan A., 2004, Ibid, p. 163 (in Armenian)

${ }^{27}$ Gr. Broutian, The Armenian Calendar, "Etchmiadzin", 1997, pp.430-433 (in Armenian)

${ }^{28}$ Gr. Broutian, The Armenian Calendar Studies, Mkhitarian Publishing House, Yerevan, 2016 (in Armenian)

${ }^{29} \mathrm{Gr}$. Broutian, Knowledge of the ancient national calendars as a means of patriotism in the publications of the Mother See, "Etchmiadzin", 2020, Issue 12, pp. 24-33 (in Armenian)
} 


\begin{tabular}{cccc} 
& Calendar Name & Period of application & Duration \\
\hline \hline a. & Protohaykian Calendar & $9000-2341 \mathrm{BC}$ & 66,5 centuries \\
b. & Haykian Calendar & $2341 \mathrm{BC}-552 \mathrm{AD}$ & 28 centuries \\
c. & Armenian Major Calendar & $552 \mathrm{AD}-20^{t h}$ century & 14 centuries \\
d. & Armenian Minor Calendar & $1112 \mathrm{AD}-19^{t h}$ century & 7,5 centuries \\
e. & Roman Calendar & $1774 \mathrm{AD}-$ to our days & 2,5 centuries \\
\hline
\end{tabular}

Table 1. The chronology of the Armenian Calendars.

"The period of the Protohaykian calendar coincides with the most ancient period of Armenian history, which was also the most significant period in terms of cultural and civilizational achievements. Such civilizational achievements as obtaining cultivated species of cereal and some other crops, the domestication of some pets, the division of the starry sky into constellations, the invention of primary writing, the invention of the mining and processing of bronze, and then iron, and more are related to this calendrical period.

Thus, based on all these, we can conclude that the Protohaykian calendar was better suited to the natural course of the (Armenian) people's lifestyle and the manifestation of his creative powers." 0

Grigor Broutian

\section{The Results of Other Studies in the Field of Cultural Astronomy}

It should be especially noted that such calendar studies include a large amount of detailed analyses from the related fields. As it was mentioned earlier, in the course of the work an attempt is made to supplement the answers to the questions of interest by obtaining and importing additional information from various spheres of Armenian folklore, ethnography and cultural heritage, that concern our issues. Such an exploration leads to new discoveries in these areas. The results of the discoveries have been published in a number of publications over the past decades. Let us review the most important ones.

1) The start of the year (Holiday of Navasard) in the Pre-Christian Armenian calendars was clarified. There are different opinions about the position of Navasard, yet, as the examination $^{31}$ of numerous calendrical, astronomical and ethnographic facts shows, the original position of Navasard was in the middle of the astronomical spring and summer, 8 days before the summer solstice. Such a position of Navasard, among many other facts, is also confirmed by the testimony of Agatangelos that Navasard was considered the "Festival of New Harvest". Indeed, in Armenia the first harvest ripens during the beginning of summer season. It's especially important to note that during these days winter wheat also grows which has been the basis of the prosperity of the Armenian farmers for thousands of years. As it was already mentioned above, in the Haykian calendar as well this day was determined by the observation of the heliacal rising of the main star of the Hayk constellation at the $10^{\text {th }}$ hour in the Aravot (Morning), which is about 1,5 hours before sunrise. Special rituals and a celebration ${ }^{32}$ were held during the holiday.

2) In the meantime, $\operatorname{cosmic}^{33}$ calendar $^{34}$ realities were revealed in many examples of Armenian folk tales. The information contained in Armenian fairy tales, first of all, provides insight into the worldview of their creators. It is in these perceptions that we find some answers to the questions that we are interested in and use them as auxiliary information to make the

\footnotetext{
${ }^{30}$ Gr. Broutian, BAO, 2018, Ibid. pp. 174-175 (in Armenian)

${ }^{31}$ Gr. Broutian, "Etchmiadzin", 1995, Issues 10-12, pp.130-153 (in Armenian)

${ }^{32}$ Gr. Broutian, "Etchmiadzin", 1992, Issues 4-5, pp.95-118 (in Armenian)

${ }^{33}$ Gr. Broutian, "Etchmiadzin", 2008, Issue 2, pp.49-66 (in Armenian). Gr. Broutian, The Understanding of Time and Space According to Armenian Fairy Tales, "Bazmavep", 2011, vol. 3-4, pp.459-484 (in Armenian)

${ }^{34}$ Gr. Broutian, "Etchmiadzin", 2009, Issue 12, pp.62-83 (in Armenian). Gr. Broutian, "Etchmiadzin", 2010, Issue 6, pp.22-44 (in Armenian)
} 
result of some "reverse problem's" solution univocal. Hayk's children can be regarded as the clearest example. To find out how many of Hayk's 10 children are sons, among other sources, we get help from tales, in which the protagonists are the king's three sons, the youngest of whom becomes the father's heir, as was the case with Hayk's youngest son Aramanyak ${ }^{35}$.

3) Numerous manifestations of astronomical and calendar realities have been revealed in the Armenian "Sasnay Tsrer" Epic poem ${ }^{36}$. The parallels between the main characters of the epic poem and the constellations of the Zodiac have been clarified. In particular, the analysis has shown that the most discussed hero's, Davids' character paralleled the Sagittarius constellation and the planet Lusntag (Jupiter). The Elder-Pisces (based on the Mesopotamian constellation A-nu-ni-tu, which is located in the current region of Pisces), Pokr (Small) Mher - Aries, Dzenov Ohan - Taurus, Sanasar and Baghdasar - Gemini, Tzran Vergo - Tzir (present Cancer Zodiac sign), Mets (Big) Mher - Leo and other comparisons are also important. These, in fact, are adjacent constellations in the Zodiac. On the basis of such realities an attempt was made to date the Epic poem. Calendar parallels ${ }^{37}$ with the celestial realities of considerable antiquity have been obtained (17700 BC). The poem's episode ${ }^{38}$ in which Tsovinar (Virgo) advises his twin sons to escape from Baghdad, taking the bright star for direction, has also been examined. The work is based on two starting points: a) only a Pole star (or very close to the North Pole) can indicate a direction, as fixed; b) based on religious beliefs (worship of the cross) the main star of the Vulture (Cygnus) constellation, Deneb ( $\alpha$ Cygnus) was taken as such. As a result, the possible date of this episode of the Epic poem has been estimated to $15500 \mathrm{BC}$. This is the time when the mentioned star was the closest to the North Pole.

4) A significant change in the classification of Armenian "Vishap" (dragon) stones has been proposed on an astronomical basis. It was generally accepted to classify these "Dragon Stones" into three types: fish-shaped, with the image of cattle (these are sometimes called bull heads) and with mixed images. With the detailed analysis of the "Vishap" stone sculptures, it was possible to distinguish their 5 types: a) with the head of a wild donkey, b) depicting a pair of storks, c) with the bull head, d) with the head of a ram and e) in the form of fish. This series, obviously, repeats a part of neighboring constellations of the zodiac: a) Cancer (wild donkey, donkey), b) Gemini, c) Taurus, d) Aries, e) Pisces respectively. Based on such correspondence, an attempt has been made ${ }^{39}$ to date these monuments astronomically. As a result, the time related to them was set 28500-19900 BC, moreover, according to the type of the "Vishap" stone classification the approximate chronology has been calculated separately. It is also important to emphasize that the imagery of the Gemini constellation has been revealed in the form of a pair of storks.

5) Based on the structure of the Armenian calendar, some circumstances about a number of calendars of neighboring nations have been explained ${ }^{40}$. In particular, the main year the ancient Roman calendar being of 10-month (304-day) long (as well as many other features) is explained ${ }^{41}$ by the direct or mediated borrowing of the Armenian Protohaykian calendar. This borrowing is also evidenced by a detailed examination ${ }^{42}$ of all the expressions

\footnotetext{
${ }^{35}$ Gr. Broutian, The Armenian Calendar, "Etchmiadzin", 1997, Chapter 2, pp.386-399 (in Armenian)

${ }^{36}$ Gr. Broutian, Some Astronomical-Cosmic Realities in the Armenian Epic "Sasnay Tsrer", Astronomical Heritage of the Middle East, ASP Conference Series, Astronomical Society of the Pasific, 2019, Vol. 520, pp. 191-198

${ }^{37} \mathrm{Gr}$. Broutian, An attempt to determine the time of an episode of the Armenian "Sasnay Tsrer" epic through astronomical realities, "Etchmiadzin", 2021, (accepted)

${ }^{38}$ Gr. Broutian, An Astronomical Attempt to Determine the Temporal Origin of an Episode of the Armenian Epic "Sasnay Tsrer" Communications of BAO, Vol. 68, Issue 1, 2021, pp. 105-113 doi:https://doi.org/10.52526/257927762021.68.1-105

${ }^{39}$ Gr. Broutian, "Etchmiadzin", 2020, Issue 4, pp.44-65 (in Armenian)

${ }^{40} \mathrm{Gr}$. Broutian, Georgian and Alouanian calendars as presented in Anania Shirakouni's Tomar (Calendar): Some problems concerning their origin and structure, Armenia and Christian Caucasus, Republican Conference devoted to the 1700-th Anniversary of adoption of Christianity in Caucasian Albania and Georgia, Yerevan, 15-16 December 2015, Papers and abstracts of papers, pp. 58-68

${ }^{41}$ Gr. Broutian, "Etchmiadzin", 2019, Issue 7, pp.64-84 (in Armenian)

${ }^{42}$ Gr.Broutian, Armen deacon Khachatryan, Certain Issues Related to the Calendar in the Holy Bible, "Bazmavep",
} 
with time-related words contained in the Book of Exhodus of the Torah. More than 20 significant similarities have been identified between the testimonies of the 7-day ritual of the main Jewish holiday (which was also the main point of the Exodus of the Jews from Egypt) and the Navasard celebrations in the Protohaykian calendar.

6) Extensive astronomical research on the Armenian megalithic monuments has begun. The comparative analysis of perhaps the most well-known and discussed monument in Armenia, the baseline structure of the "Zorats Qarer" and the arrangement of the stars of the Eagle-Cygnus constellation, has shown an almost complete correspondence in 30300-29300 BC millennial domain ${ }^{43}$. This examination differed from the previous ones as more than one baseline data were taken into account. The task was to find the time when all the dimensional data of the plan of the monument and the geographical location coincide with the equatorial coordinates of the constellation stars. Given the result, it became necessary to thoroughly research the monument from an astronomical point of view. For this purpose, within the framework of the cooperation agreement signed between the Byurakan Astrophysical Observatory (BAO) and the National University of Architecture and Construction of Armenia, a 1-week scientific expedition was carried out in July, 2020. The aim of the scientific expedition was to examine the "Zorats Qarer" monument from an astronomical point of view and obtain a complete measurement data using modern digital measuring devices (an area of about 20 ha was measured to the nearest $1 \mathrm{~mm}$ in length and an angular dimension of 10 arc seconds). A detailed examination revealed specially cut angular stones (Figure 1) and platforms, which, as it was discovered later, had special observational importance. In addition, the first results ${ }^{44}$ showed the connection of this monument with the Haykian and Protohaykian calendars discussed above. In general, the preliminary data show that the monument is multi-layered (9000 BC, $5800 \mathrm{BC}$ and $2341 \mathrm{BC}$ ). Moreover, it should be emphasized that the observational realities revealed in the monument within the mentioned dates also show the continuation of the logical sequential development of the same cultural manifestations over the millennia. These studies are currently in progress and their results will be published in the near future ${ }^{45}$. The other megalithic monument which attracted our attention during the preliminary examination, is the archaeological site near the village of Hartashen, Shirak Region. The very first observations of this monument revealed some patterns which speak of its possible connection with astronomical phenomena. To study these assumptions in depth, measurements of the Hartashen monument were needed. This work began in June 2021 within the framework of the above-mentioned cooperation agreement and it still continues.

The foregoing results, as we see in 3, 4 and 6 sub-items, refer to the events of enormous antiquity (30000-15000 BC), information about which has not reached us. However, as it was mentioned, this period preceded the beginning of the Protohaykian calendar $9000 \mathrm{BC}$. Thus, the astronomicalcalendrical notions of such antiquity probably refer to the Preliminary Calendar. Let us single out only one coincidence, which is obvious from 3 and 4 sub-items. We see almost the same span and time sequence of the constellations compared to the types of "Vishap" (dragon) stones and the main characters of the "Sasnay Tsrer" epic poem, which raises the need to answer a number of questions. The time "Zorats Qarer" megalithic monument was dated, on the other hand, also leaves many questions unanswered. All in all, we see that we are dealing with the multifaceted, millennial heritage of our ancestors that belong to the same culture. So, it is obvious that this field requires special attention, and future research with the introduction of wide opportunities is of great importance.

\footnotetext{
2008, pp. 83-100 (in Armenian)

${ }^{43}$ Malkhasyan H. A., The observation of Angegh-Vulture (Cygnus) constellation in Armenia 32000 years ago, Communications of BAO, Vol. 67, Issue 1, 2020, pp. 27-36. doi: 10.52526/25792776-2020.67.1-27

${ }^{44}$ Gr. Broutian, H. Malkhasyan, Ibid.

${ }^{45}$ Malkhasyan H.A., Some New Results of the Study of "Zorats Qarer" Megalithic Monument (Platform 2), "Bazmavep", 2021, (accepted)
} 